\title{
Temperature Effect on the Structure of Transformer Oil Based Magnetic Fluids Using Acoustic Spectroscopy
}

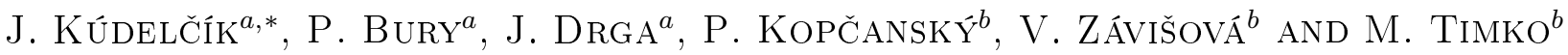 \\ ${ }^{a}$ Department of Physics, University of Žilina, Univerzitná 1, 01001 Žilina, Slovakia \\ ${ }^{b}$ Department of Magnetism, IEP SAS, Watsonova 47, 04001 Košice, Slovakia
}

\begin{abstract}
The changes in structural arrangement in transformer oil based magnetic fluids upon the effect of an external magnetic field and temperature were studied by acoustic spectroscopy. The attenuation of acoustic waves was measured as a function of an external magnetic field in the range of $0-300 \mathrm{mT}$, parallel to the direction of the field and as a function of temperature in the range of $15-35^{\circ} \mathrm{C}$ for various magnetic nanoparticles concentrations. The strong influence of the steeped magnetic field on the acoustic wave attenuation was detected and its hysteresis was observed, too. When a magnetic field is swept at a constant rate, the dominant interactions between the external magnetic field and the magnetic moment of the nanoparticles occur, leading to the aggregation of magnetic nanoparticles and clusters formation. However, the temperature of magnetic fluids has very important influence on the obtained dependences, where the mechanism of thermal motion acts against the cluster creation. The observed influences of magnetic field and temperature on the investigated magnetic liquids structure are discussed.
\end{abstract}

PACS: 75.50.Mm, 75.75.Jn

\section{Introduction}

Magnetic fluids have attracted remarkable physical properties that have recently found wide application in technology and medicine [1]. The heat transfer in electromagnetic devices such as transformers can be substantially improved by using magnetic nanoparticles suspended in transformer oil. The dielectric breakdown strength of transformer oil, however, is strongly influenced by the aggregation effects of magnetic particles and can induce electric breakdown [2, 3].

A magnetic fluid is a colloidal suspension of nanosized magnetic particles covered with a surfactant layer in a carried liquid [1]. Particles of this size are monodomain and surfactant produces entropic repulsion between them. So without an external magnetic field this cover prevents the interaction between them that may lead to their agglomeration and subsequent sedimentation. The macroscopic magnetic properties of the magnetic fluid are determined by the orientation of magnetic moments of nanoparticles and their agglomeration in the external magnetic field. In externally applied magnetic field magnetic fluid behaves as paramagnetic, magnetization increases with the field. Magnetic field can also cause that some of magnetic particles join into quasispherical objects and clusters as long as hundreds of nanometers or more $[4,5]$.

One of the useful tool to study changes in the magnetic fluid structure is based on the measurements of changes in acoustic wave attenuation $\Delta \alpha$ under the influence of an external magnetic field and the temperature of magnetic

* corresponding author; e-mail: kudelcik@fyzika.uniza.sk fluid $[4,5]$. The propagation and attenuation of acoustic waves through the magnetic fluid, suspensions of two phases - magnetic particles dispersed in a continuous carried fluid, can be used to characterize their structural properties. The interaction between the acoustic waves and the aggregated magnetic particles or clusters leads to the additional absorption of acoustic wave compared to that in the carried fluid [1,4-6].

In the present study the changes of the acoustic attenuation as a function of external magnetic field and temperature in transformer oil TECHNOL based magnetic fluids are investigated. The observed effects are analyzed and discussed.

\section{Experimental}

The block diagram of the experimental arrangement is shown in Fig. 1. The measurements of the attenuation of the acoustic wave of frequency $13.3 \mathrm{MHz}$ were carried out by a pulse method using the MATEC Pulse Modulator and Attenuation Recorder, model 7700 [7]. An acoustic wave is generated by transducer place in the thermally stabilized measuring cell $\left(1.5 \times 0.9 \times 1 \mathrm{~cm}^{3}\right.$, the temperature stabilized with an accuracy $\pm 0.2{ }^{\circ} \mathrm{C}$ ) inserted in an electromagnet. Acoustic wave propagating in magnetic fluid undergoes a multiple reflection from the transducers and its subsequent echoes are fed to the MATEC receiver. The signal from the MATEC, which is proportional to the changes of the acoustic attenuation in the magnetic fluid, was recorded and evaluated by a computer [8]. Computer also controlled current source for the electromagnet.

The magnetic fluids used in experiments consisted of magnetite particles $\left(\mathrm{FeO} \cdot \mathrm{Fe}_{2} \mathrm{O}_{3}\right)$, the mean diameter 


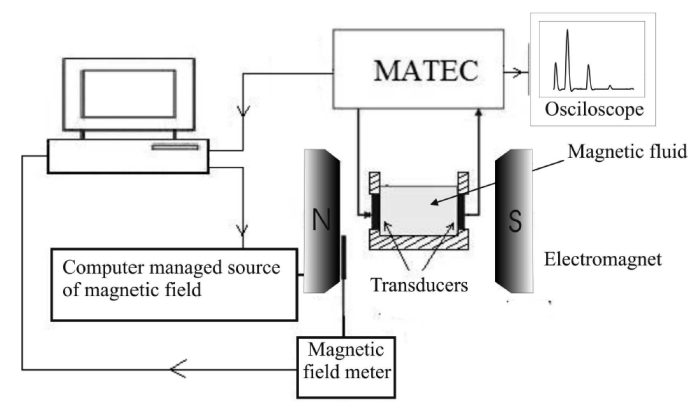

Fig. 1. Experimental setup.

$D=11.1 \mathrm{~nm}$, coated with oleic acid as a surfactant, dispersed in transformer oil TECHNOL. The basic properties of this magnetic fluid, such as the density, saturation magnetization and volume fraction have values $0.882 \mathrm{~g} / \mathrm{cm}^{3}, 0.917 \mathrm{~g} / \mathrm{cm}^{3}, 0.976 \mathrm{~g} / \mathrm{cm}^{3}$ and $4.7 \mathrm{mT}$, $9.5 \mathrm{mT}, 14.1 \mathrm{mT}$ and $1.0,2.5$ and $5.0 \%$, respectively.

\section{Experimental results and discussion}

The attenuation of acoustic wave depends on the magnetic field intensity, the rate of its changes and the temperature of magnetic fluid. Figure 2 presents the attenuation change as a function of magnetic field $\boldsymbol{B}$ for the same sweep rate, which is parallel to $\boldsymbol{k}$, for $1.0 \%$ magnetic fluid (MF) where magnetic field was linearly increased $(2.2 \mathrm{mT}$ per minute) to maximum value $100 \mathrm{mT}(\mathbf{\bullet}), 200 \mathrm{mT}(\bullet)$ and $300 \mathrm{mT}(\mathbf{\Delta})$, respectively. The magnetic field after reaching the maximum value and 1 min pause decreased at the same rate (see Figs. 2, 3).

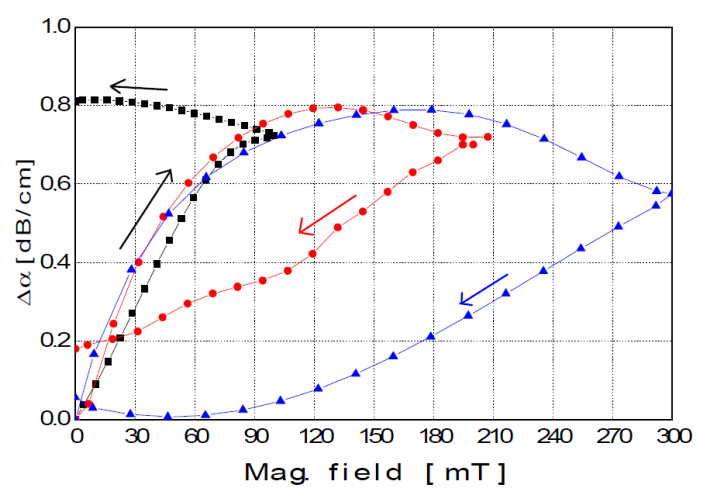

Fig. 2. Changes of the attenuation of acoustic wave in $1.0 \% \mathrm{MF}$ for frequency $f=13.3 \mathrm{MHz}$ and temperature $20{ }^{\circ} \mathrm{C}$ in the external magnetic field versus the magnetic field, for different maximum values at constant sweep rate $2.2 \mathrm{mT} / \mathrm{min}(\bullet 100 \mathrm{mT}, \bullet 200 \mathrm{mT}, \boldsymbol{\Delta} 300 \mathrm{mT})$.

These results show a strong influence of the magnetic field on the value of acoustic attenuation. With increasing magnetic field, the acoustic absorption increases, because the interactions between the magnetic field and the magnetic moment of the nanoparticles leads to aggregation of particles and clusters formation (structures

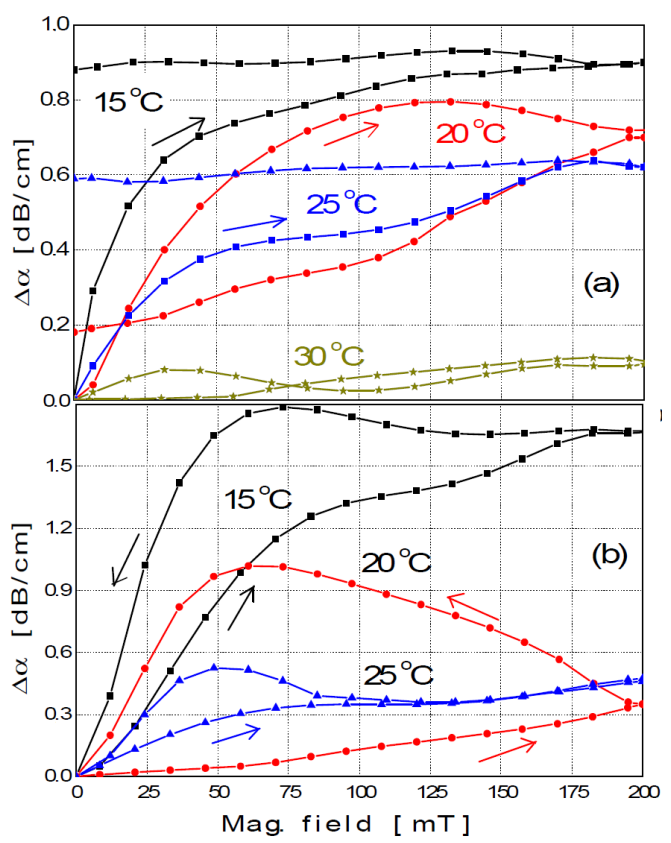

Fig. 3. The temperature dependence of changes attenuation in external magnetic field for (a) $1.0 \%$ (sweep rate $2.2 \mathrm{mT} / \mathrm{min}$ ) and (b) $5.0 \% \mathrm{MF}$ (sweep rate $5 \mathrm{mT} / \mathrm{min}$ ) for frequency $f=13.3 \mathrm{MHz}\left(15^{\circ} \mathrm{C}\right.$, - $20{ }^{\circ} \mathrm{C}, \Delta 25^{\circ} \mathrm{C}, * 30^{\circ} \mathrm{C}$ ).

as long as hundreds of nanometers [5]). Between 100$200 \mathrm{mT}$ the acoustic attenuation is practically saturated. However, the mutual connection of clusters at higher magnetic field results in their lower concentration and resulting decrease of acoustic absorption. The character of attenuation changes continues also with decreasing magnetic field as a result of continuation of beginning processes. However, the character of attenuation changes with decreasing magnetic field depends on the achieved value of magnetic field $(100,200$, or $300 \mathrm{mT})$. This is the reason that the changes of the acoustic attenuation show a hysteresis $[1,4,8]$. This effect can be described by existence of clusters, whose lifetimes after their formation is longer than time of decrease of the magnetic fluid, so that the structure does not return to the initial state immediately after the magnetic field has been removed.

The acoustic attenuation was investigated for all concentrations of magnetic fluids in the temperature range of $15-30^{\circ} \mathrm{C}$. The results indicate a significant effect of the temperature on the acoustic attenuation. For $1 \% \mathrm{MF}$ and to the temperature $25^{\circ} \mathrm{C}$ (Fig. 3a), the measurement of the acoustic attenuation shows the largest changes originating from the process of cluster creation and shows also higher hysteresis. At these temperatures the creation of cluster is more effective because the Brown thermal motion is not so effective to destroy the clusters [5]. At the temperature $30^{\circ} \mathrm{C}$ the structure of clusters disappears and the majority of magnetic particles seem to be practically free. In the case of $5 \% \mathrm{MF}$ the largest changes of attenuation and higher hysteresis is observed only at 
lower temperature (Fig. $3 \mathrm{~b}, 15^{\circ} \mathrm{C}$ ). At the temperature $20^{\circ} \mathrm{C}$ the development of change of acoustic attenuation with increase of magnetic field is no so marked (Fig. 3b). The absorption coefficient still increases at the decrease of magnetic field until $60 \mathrm{mT}$, the length of clusters still increases. At this concentration of magnetic fluid the thermal motion destroyed clusters already at the temperature $25^{\circ} \mathrm{C}$. The influence of magnetic field on the acoustic attenuation happens small and as follows from our results the majority of the particles are not involved in the cluster structures. The stability of such medium that is the lack of tendency towards investigated cluster formation increases in higher temperatures. The magnetization state and the corresponding acoustic properties of investigated magnetic fluids depend strongly on temperature. This is the result of interaction of acoustic wave with magnetic particles or clusters.

In a magnetic fluid not subjected to an external magnetic field, the acoustic attenuation, $\alpha_{\exp }$, is firstly related to the dynamic viscosity, $\eta_{\mathrm{S}}$, and volume viscosity, $\eta_{\mathrm{V}}[5]$ :

$$
\alpha=\frac{2 \pi^{2} f^{2}}{\rho c^{3}}\left(\frac{4}{3} \eta_{\mathrm{S}}+\eta_{\mathrm{V}}\right),
$$

where $\rho$ is the density, $c$ is the velocity of the ultrasonic wave and $f$ is the frequency of the ultrasonic wave. The additional terms of attenuation coefficient originate from rotational motion of clusters, but these terms are negligible without external magnetic field. Because of a strong temperature dependence of the viscosity (the Arrhenius formula), it can be expected that with increasing temperature the acoustic attenuation will decrease.

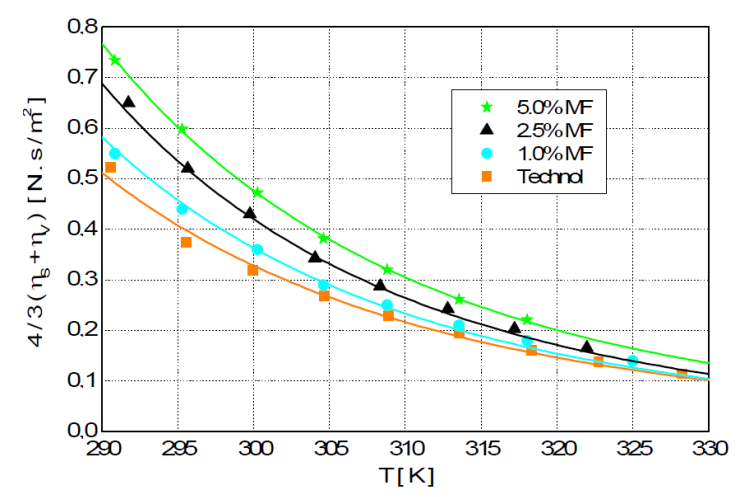

Fig. 4. The Arrhenius fit of the temperature dependences of $(4 / 3) \eta_{\mathrm{S}}+\eta_{\mathrm{V}}$ for the various concentration of magnetic fluid, without the magnetic field.

The temperature dependence of the term $(4 / 3) \eta_{\mathrm{S}}+\eta_{\mathrm{V}}$ enable the fit with the Arrhenius function

$$
\left(\frac{4}{3} \eta_{\mathrm{S}}+\eta_{\mathrm{V}}\right)=f(T)=A \exp \left(\frac{B}{T}\right)
$$

where $A$ and $B$ are coefficients dependent on the proper- ties of magnetic fluids. As follows from Fig. 4 the coefficient of viscosity decreases with increasing temperature [9]. Also the viscosity coefficient increases with the concentration of magnetic nanoparticles in magnetic fluid.

\section{Conclusion}

This study was focused on both the magnetic field and temperature induced changes of the structure of magnetic nanoparticles in the transformer oil based magnetic fluids. The strong influence of magnetic field and temperature on the structures was detected using acoustic spectroscopy. The observed behavior of the acoustic attenuation validated the process of aggregation of the magnetic nanoparticles into clusters on the one side and the role of thermal motion on their disintegration. The character of the temperature changes of acoustic attenuation can be related to the increasing value of the viscosity coefficient at lower temperatures when the number at magnetic domains involved in the structural of clusters increases.

\section{Acknowledgments}

This work was supported by projects VEGA 2/0077/09, 1/0548/09 and MNT ERA Net MAFINCO. The authors wish to thank also for the support to the R\&D operational program Centre of excellence of power electronics systems and materials for their components. The project is funded by European Community, ERDF - European regional development fund.

\section{References}

[1] A. Józefczak, J. Magn. Magn. Mater. 240-244, 293 (2005).

[2] P. Kopčanský, K. Marton, M. Koneracká, M. Timko, I. Potočová, J. Magn. Magn. Mater. 415-418, 289 (2005).

[3] J. Kúdelčík, P. Bury, V. Závišová, M. Timko, P. Kopčanský, Communication 34-37, 2 (2010).

[4] A. Józefczak, A. Skumiel, M. Łabowski, J. Magn. Magn. Mater. 356-359, 252 (2002).

[5] A. Skumiel, J. Phys. D, Appl. Phys. 3073-3079, 37 (2004).

[6] T. Sawada, H. Nishiyama, T. Tabata, J. Magn. Magn. Mater. 186-188, 252 (2002).

[7] P. Bury, P. Hockicko, I. Jamnický, Adv. Mater. Res. 111-116, 39 (2008).

[8] J. Kúdelčík, P. Bury, V. Závišová, M. Timko, P. Kopčanský, Acta Electrotechn. Inform. 90-92, 10 (2010).

[9] T. Hornowski, A. Skumiel, M. Labowski, Mol. Quant. Acoust. 41-46, 24 (2003). 